\title{
Chronic Kidney Disease Requiring Healthcare Services: A New Approach to Evaluate Epidemiology of Renal Disease
}

\author{
Gianluca Trifirò, ${ }^{1}$ Janet Sultana, ${ }^{1}$ Francesco Giorgianni, ${ }^{1}$ Ylenia Ingrasciotta, ${ }^{1}$ \\ Michele Buemi, ${ }^{1}$ Marco Muscianisi, ${ }^{1}$ Daniele Ugo Tari, ${ }^{2}$ Margherita Perrotta, ${ }^{2}$ \\ Valeria Canale, ${ }^{1}$ Vincenzo Arcoraci, ${ }^{1}$ and Domenico Santoro ${ }^{1}$ \\ ${ }^{1}$ Department of Clinical and Experimental Medicine, University of Messina and Policlinico Universitario, \\ Via Consolare Valeria, 98125 Messina, Italy \\ ${ }^{2}$ Caserta-1 Local Health Service, 81100 Caserta, Italy \\ Correspondence should be addressed to Gianluca Trifirò; trifirog@unime.it
}

Received 5 February 2014; Accepted 14 September 2014; Published 20 November 2014

Academic Editor: Lorenzo Cavagna

Copyright (C) 2014 Gianluca Trifirò et al. This is an open access article distributed under the Creative Commons Attribution License, which permits unrestricted use, distribution, and reproduction in any medium, provided the original work is properly cited.

\begin{abstract}
Background. Screening-based CKD estimates may not provide a sufficient insight into the impact of CKD on the use of healthcare resources in clinical practice. The aim of this study was to evaluate the epidemiology of "medicalized" CKD, that is, CKD requiring healthcare services, in an outpatient setting. Design, Setting, Participants, and Measurements. This is a retrospective, longitudinal population-based study conducted in a large general practice setting in Southern Italy (Caserta) using a healthcare database. Over 2006-2011, all patients with a CKD diagnosis, either through CKD-related indications of use associated with drug prescriptions or through CKD-related hospital discharge diagnoses/procedures, were identified using this database. The prevalence of "medicalized" CKD in the general population of Caserta was estimated by age, gender, and calendar year. Results. Overall, 1,989 (1.3\%) patients with a diagnosis of CKD were identified from 2006-2011 in the Caserta general population. The one year prevalence increased from $0.9 \%$ in 2006 to $1.6 \%$ in 2011, which is much lower compared to previous screening-based studies. The prevalence was slightly higher in males and increased significantly with advancing age (in 2011, $0.2 \%$ in $\leq 44$ years old versus $9.2 \%$ in $>80$ years old). Conclusions. The findings of this study suggest that, in the general population, the prevalence of "medicalized" CKD is lower compared to the screening-based CKD prevalence.
\end{abstract}

\section{Background}

The number of patients worldwide with chronic kidney disease (CKD) is continuously increasing. Although CKD has been a somewhat scientifically neglected chronic noncommunicable disease [1], the global burden of CKD has been found to increase year after year. The main driving factors behind this are the increasingly aged global population [2] and the worldwide epidemic of type 2 diabetes mellitus [3].

Prevalence studies have a particularly relevant role in healthcare planning since global healthcare resources are limited, while healthcare needs are constantly increasing [4]. Several epidemiological studies such as PREVEND (The Netherlands) [5], EPIRCE (Spain) [6], HUNT (Norway) [7], NHANES III (USA) [8], and INCIPE (Italy) [9] have explored the prevalence of the various stages of CKD in different countries. Depending upon the method for CKD identification or formula for estimating the glomerular filtration rate (GFR), the race (Caucasian, Afro-American or Asian, etc.), or the setting, the prevalence of different CKD stages is often comparable in these studies, ranging from 5.1 to $7.0 \%$ for stages 1 and 2 combined, from 4.5 to $5.3 \%$ for stage 3 , and much lower for stages 4 and 5 , from 0.1 to $0.4 \%$ (Table 1). Recently, data coming from Italian (CARHES) [10] and Chinese [11] studies that examined small samples of the general population found that prevalence of CKD is lower as compared to other countries, especially concerning CKD 3-5 stages. However, the real impact of CKD on healthcare systems has not been well determined because CKD studies generally do not specifically consider CKD cases that are allocated healthcare resources. In particular, the attention should be focused not only on the screening-based 


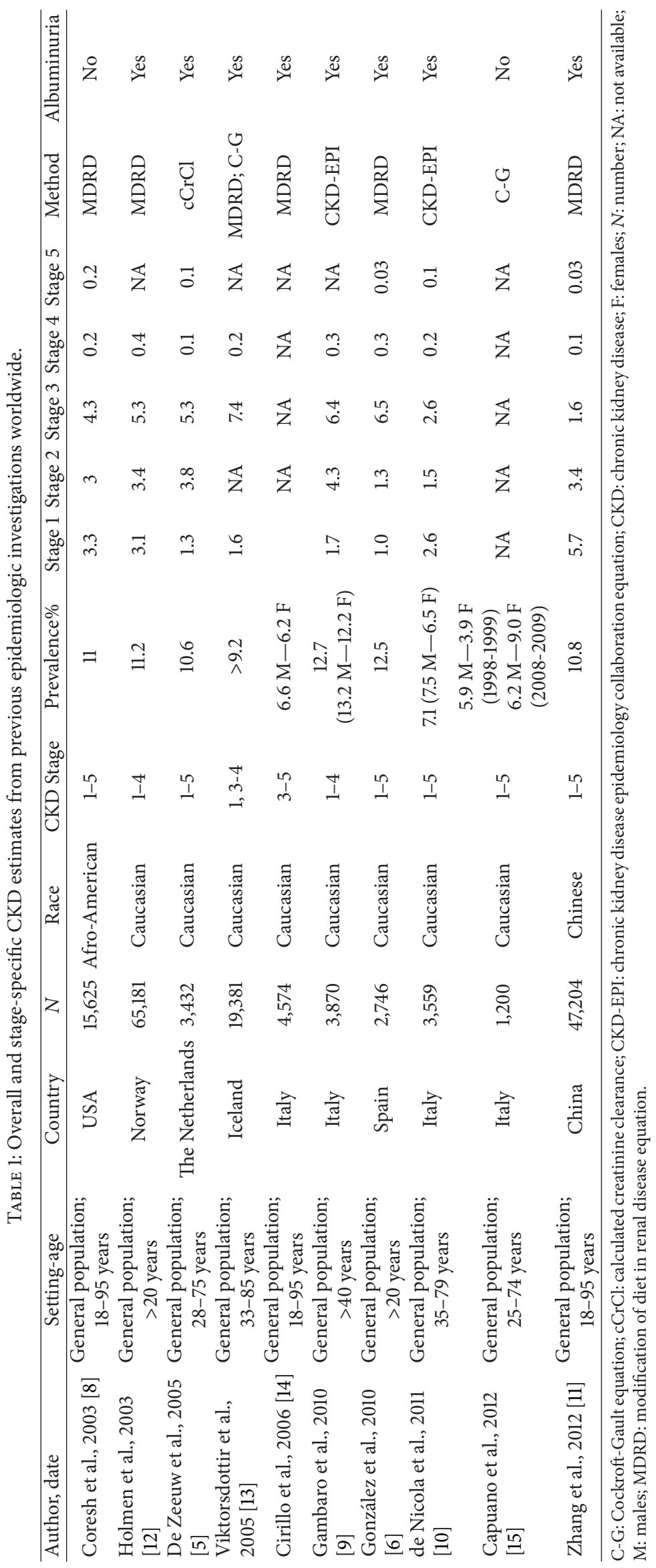


prevalence of CKD, but on the CKD populations that require the use of resources of the healthcare systems directly. For this reason, we explored the prevalence of CKD requiring drug prescriptions, hospital admissions, and procedures, that is, what we termed "medicalized" CKD, in a general population of Southern Italy using a claims database.

\section{Methods}

Data was extracted from the Arianna database during the years 2004-2011. This database was set up by the Caserta Local Health Unit in Southern Italy in the year 2000 and currently contains information on a population of 158,510 inhabitants $(20 \%$ of population from Caserta catchment area), who are registered in the list of 123 general practitioners (GPs). During their daily routine care, GPs record and transfer anonymous patient clinical data to a central database through dedicated software.

The Arianna database contains data concerning all the drug prescriptions (and related indication of use) which are reimbursed by the National Health Service (NHS). This data can be linked to hospital discharge admissions and other registries through a unique patient identifier. Information on drugs is coded according to the Anatomical Therapeutic Chemical classification system (ATC), while indications for use and hospital discharge diagnoses/procedures are coded by the ninth edition of International Classification of Diseases-Clinical Modification (ICD-9 CM). Quality checks on the data are routinely carried out. Arianna database has been previously demonstrated as a valid source for epidemiological research [16-19].

We identified CKD patients, searching for the following specific renal diseases-related codes among either primary/secondary causes of hospital admission or indication of use associated to prescribed drugs: 250.4 (diabetes with renal manifestations), 285.21 (anemia in chronic kidney disease), $583^{*}$ (nephritis and nephropathy, not specified as acute or chronic), 585* (chronic kidney disease), 586* (renal failure, unspecified), 403* (hypertensive chronic kidney disease), and $404^{*}$ (hypertensive heart and chronic kidney disease). Patients with ICD-9 codes $583^{*}$ or $586^{*}$ were considered as CKD patients only if these codes were repeated more than twice to prevent misclassifying acute renal disease as CKD. We identified CKD also in the presence of multiple registration of renal dialysis among procedures over time.

We calculated the one year prevalence of "medicalized" CKD, overall, by age groups and by sex, over the years 20062011, with the years 2004-2005 being considered as run-in period to characterize the patients. For each observation year, the CKD prevalence was calculated by dividing the number of patients with CKD diagnosis by the number of subject who were registered in the GPs' lists.

\section{Results}

Out of almost 160,000 persons from a general population of Southern Italy in the years 2006-2011, we identified 1,989 patients $(1.3 \%)$ with a diagnosis of CKD requiring drug prescriptions for renal disease-related indications of use or
TABLE 2: Healthcare resources used for the identification of first CKD diagnosis.

\begin{tabular}{|c|c|}
\hline Healthcare resources & $\begin{array}{l}\text { CKD patients } \\
N=1,989(\%)\end{array}$ \\
\hline CKD-related hospital discharge diagnoses* & $1,151(57.9)$ \\
\hline Primary diagnosis & $258(13.0)$ \\
\hline Secondary diagnosis & $867(43.6)$ \\
\hline Procedures & $39(2.0)$ \\
\hline $\begin{array}{l}\text { CKD-related conditions as indication of use } \\
\text { for drug prescriptions }\end{array}$ & $838(42.1)$ \\
\hline Allopurinol & $213(10.7)$ \\
\hline Furosemide & $94(4.7)$ \\
\hline Polystyrene sulfonate & $63(3.2)$ \\
\hline Calcitriol & $56(2.8)$ \\
\hline Ramipril & $29(1.5)$ \\
\hline Metformin and sulfonamides & $28(1.4)$ \\
\hline Acetylsalicylic acid & $24(1.2)$ \\
\hline Metformin & $22(1.1)$ \\
\hline Electrolytes solutions & $19(1.0)$ \\
\hline Glimepiride & $16(0.8)$ \\
\hline Ferrous sulfate & $12(0.6)$ \\
\hline Torasemide & $12(0.6)$ \\
\hline Amlodipine & $11(0.6)$ \\
\hline Simvastatin & $10(0.5)$ \\
\hline Bisoprolol & $9(0.5)$ \\
\hline Nebivolol & $9(0.5)$ \\
\hline Losartan & $9(0.5)$ \\
\hline Atorvastatin & $9(0.5)$ \\
\hline Spironolactone & $8(0.4)$ \\
\hline Ramipril plus diuretics & $8(0.4)$ \\
\hline Others** & $177(33.2)$ \\
\hline
\end{tabular}

${ }^{*}$ Categories of discharge diagnoses and procedures are not mutually exclusive as in some patients CKD-related discharge diagnosis and procedures may have been registered at the same time.

** Other drugs which account for less than $0.4 \%$ of first CKD diagnosis identification.

hospital admission/procedures (Table 2). Specifically, 1,151 (58\%) CKD patients were identified through primary or secondary hospital discharge diagnoses/procedures, while $838(42 \%)$ were identified through prescriptions issued for CKD-related indications. Of the latter, 213 (11\%) patients were prescribed allopurinol, 94 (4.7\%) furosemide, 63 (3.2\%) polystyrene sulfonate, and $56(2.8 \%)$ calcitriol with a CKDrelated indication of use, as defined by ICD-9 codes.

The one year prevalence of CKD increased over the study years from $0.9 \%$ in 2006 to $1.6 \%$ in 2011 (Figure 1). In general, the prevalence was slightly higher in males (males/females: 1.1) and increased significantly with advancing age from $0.2 \%$ in $<45$ years old patients to $9.2 \%$ in patients 80 years and over in 2011 (Figure 2). While the prevalence of CKD in patients over 80 years increased dramatically over the observation period, from 3.9 to 9.2 cases per 100 inhabitants from 2006 to 


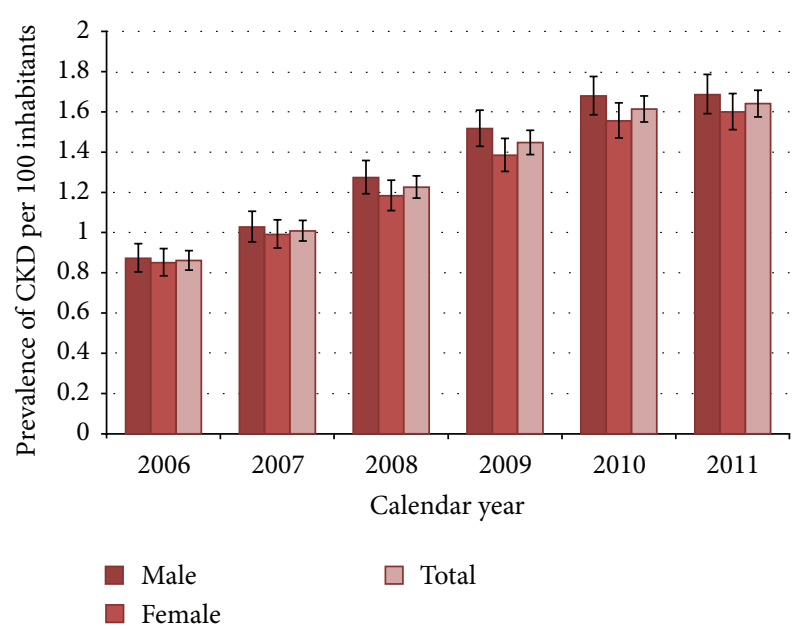

FIGURE 1: Annual prevalence (\%) of "medicalized" chronic kidney disease in general population of Caserta in the years 2006-2011, overall, and stratified by sex. The bars on the columns represent the $95 \%$ confidence intervals for proportions.

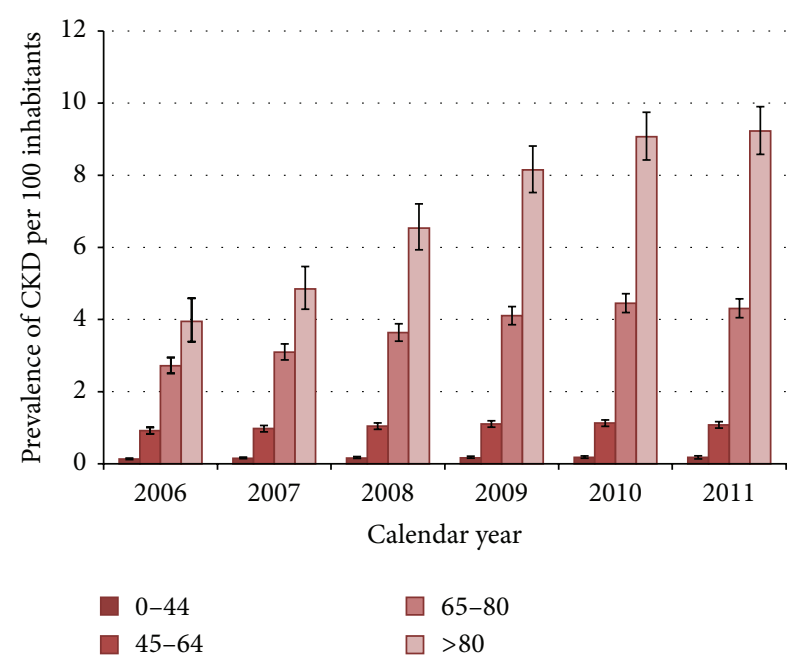

FIgURE 2: Annual prevalence (\%) of "medicalized" chronic kidney disease in the Caserta general population in the years 2006-2011, stratified by age groups. The bars on the columns represent the $95 \%$ confidence intervals for proportions.

2011, the prevalence of CKD in the other age groups remained much more stable over the years, resulting in a widening gap between CKD patients over 80 and patients under 80 .

\section{Discussion}

Several investigations have previously explored the epidemiology of CKD, mostly through screening of the general population or retrospective evaluation of GPs' electronic medical records, reporting heterogeneous results. The difference in CKD prevalence documented in various studies may be related to different methods of estimated creatinine clearance $(\mathrm{eCrCl}) \mathrm{MDRD}$ (modification of diet in renal disease equation) as compared to CKD-EPI (chronic kidney disease epidemiology collaboration equation) [20]. It is known that CKD-EPI produces higher GFR and lower CKD estimates [21]. Another reason for the observed differences in CKD prevalence is the fact that some studies screened specifically frailer target groups, such as patients with diabetes and hypertension or elderly populations, leading to an overestimation of the true CKD prevalence in the general population [22]. In the United Kingdom, patients are selected for CKD screening programs using an existing laboratory database. With this approach only patients with elevated serum creatinine or eGFR $<60 \mathrm{~mL} / \mathrm{min}$ are selected, thus precluding the identification of CKD patients at early stages (1 and 2) [23]. A better way of identifying those patients would be to select them by screening urine for a positivity using a dipstick test or using the albumin/creatinine ratio (ACR) [24]. Data from the third National Health and Nutrition Examination Survey showed that up to $11 \%$ of the general adult population could have some degree of CKD, which consisted of more than 8 million individuals with glomerular filtration rates of less than $60 \mathrm{~mL}$ per minute [8]. However, all the abovementioned screening studies, which are based on single measurement of serum creatinine or $\mathrm{eClCr}$, are subject to variation owing to differences in calibration systems across different laboratories [25].

More importantly, the epidemiology of CKD based on screening studies of small samples of the general population and retrospective evaluation of GPs' records do not allow the evaluation of the impact on actual use of healthcare services of CKD patients. The assessment of disease burden by quantifying "medicalized CKD" in combination with traditional screening-based methods may allow more accurate healthcare cost estimations for health care providers as "medicalized" CKD estimates are based on the actual use of healthcare resources.

The approach used in this study may be considered innovative. The prevalence of CKD was estimated on the basis of use of healthcare services such as CKD-related hospital admissions/procedures and drug prescriptions issued for CKD-related indications. The overall estimated prevalence of CKD in our study is significantly lower as compared to previous epidemiologic investigations. The patients identified using the claims database are likely to be mostly affected by CKD stages 4 and 5 and, to a lesser extent, 3 . In line with this hypothesis, our estimates are rather comparable to other studies when restricting the analysis to those CKD stages.

Some limitations warrant caution. Information on indication of use related to drugs being dispensed directly in hospital (e.g., epoetin, paricalcitol) was not available in Arianna database, which may have slightly underestimated the prevalence of "medicalized" CKD. However, data on the use of CKD-related hospital admissions/procedures is linked to the Arianna database, minimizing the likelihood of such an underestimation. Despite the existence of 5-digit CKD stagespecific ICD-9 codes, CKD was mostly coded using nonstage-specific 3-digit codes in the Arianna database, thus preventing the evaluation of the prevalence of CKD for each stage. 
Another potential limitation in the estimation of CKD prevalence by identifying "medicalized CKD" is that ICD codes and drug prescription-guided CKD diagnosis may be influenced by CKD awareness. As a study conducted in the same geographical area suggests, awareness of CKD is very low among patients and general practitioners [26]. Indeed, the fact that CKD prevalence in our study almost doubled from 2006 to 2011 in a small catchment area may indirectly indicate an increased awareness in assigning CKD codes at hospital discharge or a more liberal prescription of drugs for CKD-related indications, rather than an effective increase in the prevalence of CKD. Nevertheless, this is still CKD which impacts on healthcare resources. Finally, the small size of the catchment area also represents a limitation where generalizability is concerned.

In conclusion, the main results of this study demonstrate that within the general population the prevalence of CKD requiring use of healthcare resources is lower as compared to the overall screening-based CKD prevalence. From a public health perspective, the epidemiologic evaluation of CKD requiring the use of healthcare resources; that is, "medicalized" CKD may be, in addition to traditional screeningbased CKD epidemiology, valuable in providing a perspective useful to the allocation of healthcare budgets.

\begin{tabular}{|c|c|}
\hline \multicolumn{2}{|c|}{ Abbreviations } \\
\hline ACR: & Albumin/creatinine ratio \\
\hline ATC: & $\begin{array}{l}\text { Anatomical Therapeutic Chemical } \\
\text { classification system }\end{array}$ \\
\hline CARHES: & $\begin{array}{l}\text { Cardiovascular risk in Renal Patients of } \\
\text { the Italian Health Examination Survey }\end{array}$ \\
\hline CKD: & Chronic kidney disease \\
\hline CKD-EPI: & $\begin{array}{l}\text { Chronic Kidney Disease Epidemiology } \\
\text { Collaboration equation }\end{array}$ \\
\hline eCrCl: & Estimated creatinine clearance \\
\hline EPIRCE: & $\begin{array}{l}\text { Estudio Epidemiológico de la Insuficiencia } \\
\text { Renal en España }\end{array}$ \\
\hline GFR: & Glomerular filtration rate \\
\hline HUNT: & Helseundersøkelsen i Nord-Trøndelag \\
\hline ICD-9 CM: & $\begin{array}{l}\text { International Classification of Diseases - } \\
\text { Clinical Modification }\end{array}$ \\
\hline INCIPE: & $\begin{array}{l}\text { Initiative on Nephropathy, of relevance to } \\
\text { public health, which is chronic, possibly in } \\
\text { its initial stages, and carries a potential } \\
\text { risk of major clinical end-points }\end{array}$ \\
\hline MDRD: & $\begin{array}{l}\text { Modification of diet in renal disease } \\
\text { equation }\end{array}$ \\
\hline NHANES III: & National Health and Nutrition \\
\hline & Examination Survey \\
\hline NHS: & National Healthcare Service \\
\hline PREVEND: & $\begin{array}{l}\text { Prevention of Renal and Vascular } \\
\text { End-Stage Disease Intervention Trial. }\end{array}$ \\
\hline
\end{tabular}

\section{Conflict of Interests}

The authors declare that they have no competing interests.

\section{Authors' Contribution}

Gianluca Trifirò conceived the study and contributed to the study design, data analysis, interpretation of data, and the drafting of the paper. Janet Sultana participated in the interpretation of data and drafting of the paper. Francesco Giorgianni carried out the data analysis. Ylenia Ingrasciotta participated in the data analysis and in the interpretation of data. Michele Buemi, MarcoMuscianisi, Daniele Ugo Tari, Margherita Perrotta, and Valeria Canale contributed to the drafting of the paper. Vincenzo Arcoraci was involved in the acquisition of data and in the drafting of the paper. Domenico Santoro was involved in the study design, interpretation of data, and drafting of the paper. All authors read and approved the final paper.

\section{References}

[1] C. Zoccali, A. Kramer, and K. J. Jager, "Chronic kidney disease and end-stage renal disease- a review produced to contribute to the report 'the status of health in the European union: towards a healthier Europe"' NDT Plus, vol. 3, no. 3, pp. 213-224, 2010.

[2] "United States Renal Data System: annual data report: incidence and prevalence of ESRD," American Journal of Kidney Diseases, vol. 42, supplement 5, pp. S37-S173, 2003.

[3] H. King, R. E. Aubert, and W. H. Herman, "Global burden of diabetes, 1995-2025: prevalence, numerical estimates, and projections," Diabetes Care, vol. 21, no. 9, pp. 1414-1431, 1998.

[4] A. M. El Nahas and A. K. Bello, "Chronic kidney disease: the global challenge," The Lancet, vol. 365, no. 9456, pp. 331-340, 2005.

[5] D. De Zeeuw, H. L. Hillege, and P. E. De Jong, "The kidney, a cardiovascular risk marker, and a new target for therapy," Kidney International, Supplement, vol. 68, no. 98, pp. S25-S29, 2005.

[6] A. O. González, A. de Francisco, P. Gayoso, and F. García, "Prevalence of chronic renal disease in Spain: results of the EPIRCE study," Nefrologia, vol. 30, no. 1, pp. 78-86, 2010.

[7] H. Hallan, S. Romundstad, K. Kvenild, and J. Holmen, "Microalbuminuria in diabetic and hypertensive patients and the general population," Scandinavian Journal of Urology and Nephrology, vol. 37, no. 2, pp. 151-158, 2003.

[8] J. Coresh, B. C. Astor, T. Greene, G. Eknoyan, and A. S. Levey, "Prevalence of chronic kidney disease and decreased kidney function in the adult US population: third National Health and Nutrition Examination Survey," The American Journal of Kidney Diseases, vol. 41, no. 1, pp. 1-12, 2003.

[9] G. Gambaro, T. Yabarek, M. S. Graziani et al., "Prevalence of CKD in Northeastern Italy: results of the INCIPE study and comparison with NHANES," Clinical Journal of the American Society of Nephrology, vol. 5, no. 11, pp. 1946-1953, 2010.

[10] L. de Nicola, C. Donfrancesco, R. Minutolo et al., "Epidemiology of chronic kidney disease in Italy: current state and contribution of the CARHES study," Giornale Italiano di Nefrologia, vol. 28, no. 4, pp. 401-407, 2011.

[11] L. Zhang, F. Wang, L. Wang et al., "Prevalence of chronic kidney disease in China: a cross-sectional survey," The Lancet, vol. 379, no. 9818, pp. 815-822, 2012.

[12] J. Holmen, K. Midthjell, O. Kruger et al., “The Nord-Trondelag Health Study 1995-97 (HUNT 2): objectives, contents, methods and participation," Norsk Epidemiologi, no. 13, pp. 19-32, 2003. 
[13] O. Viktorsdottir, R. Palsson, M. B. Andresdottir, T. Aspelund, V. Gudnason, and O. S. Indridason, "Prevalence of chronic kidney disease based on estimated glomerular filtration rate and proteinuria in Icelandic adults," Nephrology Dialysis Transplantation, vol. 20, no. 9, pp. 1799-1807, 2005.

[14] M. Cirillo, M. Laurenzi, M. Mancini, A. Zanchetti, C. Lombardi, and N. G. de Santo, "Low glomerular filtration in the population: prevalence, associated disorders, and awareness," Kidney International, vol. 70, no. 4, pp. 800-806, 2006.

[15] V. Capuano, N. Lamaida, M. I. Borrelli et al., "Chronic kidney disease prevalence and trends (1998-2008) in an area of southern Italy. The data of the VIP project," Giornale Italiano di Nefrologia: Organo Ufficiale Della Società Italiana di Nefrologia, vol. 29, no. 4, pp. 445-451, 2012.

[16] N. Piacentini, G. Trifiró, M. Tari, S. Moretti, and V. Arcoraci, "Statin-macrolide interaction risk: a population-based study throughout a general practice database," European Journal of Clinical Pharmacology, vol. 61, no. 8, pp. 615-620, 2005.

[17] G. Trifirò, S. Corrao, M. Alacqua et al., "Interaction risk with proton pump inhibitors in general practice: significant disagreement between different drug-related information sources," British Journal of Clinical Pharmacology, vol. 62, no. 5, pp. 582590, 2006.

[18] M. Alacqua, G. Trifirò, L. Cavagna et al., "Prescribing pattern of drugs in the treatment of osteoarthritis in Italian general practice: the effect of rofecoxib withdrawal," Arthritis Care and Research, vol. 59, no. 4, pp. 568-574, 2008.

[19] A. Oteri, G. Trifirò, M. S. Gagliostro et al., "Prescribing pattern of anti-epileptic drugs in an Italian setting of elderly outpatients: a population-based study during 2004-07," British Journal of Clinical Pharmacology, vol. 70, no. 4, pp. 514-522, 2010.

[20] A. S. Levey, L. A. Stevens, C. H. Schmid et al., "A new equation to estimate glomerular filtration rate," Annals of Internal Medicine, vol. 150, no. 9, pp. 604-612, 2009.

[21] J. L. Carter, P. E. Stevens, J. E. Irving, and E. J. Lamb, “Estimating glomerular filtration rate: comparison of the CKD-EPI and MDRD equations in a large UK cohort with particular emphasis on the effect of age," QJM: An International Journal of Medicine, vol. 104, no. 10, Article ID hcr077, pp. 839-847, 2011.

[22] S. I. Hallan, K. Dahl, C. M. Oien et al., "Screening strategies for chronic kidney disease in the general population: follow-up of cross sectional health survey," British Medical Journal, vol. 333, no. 7577, pp. 1047-1050, 2006.

[23] N. Drey, P. Roderick, M. Mullee, and M. Rogerson, "A population-based study of the incidence and outcomes of diagnosed chronic kidney disease," The American Journal of Kidney Diseases, vol. 42, no. 4, pp. 677-684, 2003.

[24] H. L. Hillege, W. M. T. Janssen, A. A. A. Bak et al., "Microalbuminuria is common, also in a nondiabetic, nonhypertensive population, and an independent indicator of cardiovascular risk factors and cardiovascular morbidity," Journal of Internal Medicine, vol. 249, no. 6, pp. 519-526, 2001.

[25] J. Coresh, G. Eknoyan, A. S. Levey, C. M. Clase, A. X. Garg, and B. A. Kiberd, "Estimating the prevalence of low glomerular filtration rate requires attention to the creatinine assay calibration (multiple letters)," Journal of the American Society of Nephrology, vol. 13, no. 11, pp. 2811-2816, 2002.

[26] R. Minutolo, L. De Nicola, G. Mazzaglia et al., "Detection and awareness of moderate to advanced CKD by primary care practitioners: a cross-sectional study from Italy," The American Journal of Kidney Diseases, vol. 52, no. 3, pp. 444-453, 2008. 


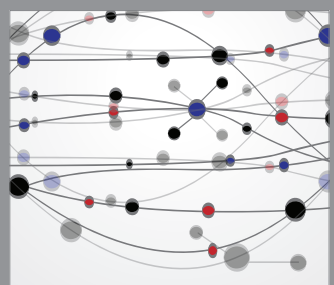

The Scientific World Journal
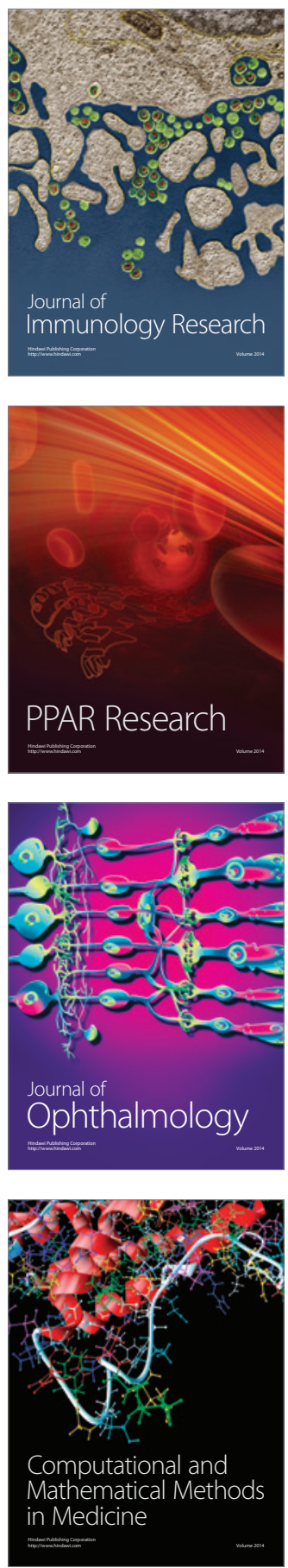

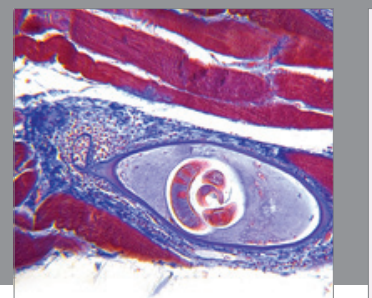

Gastroenterology

Research and Practice
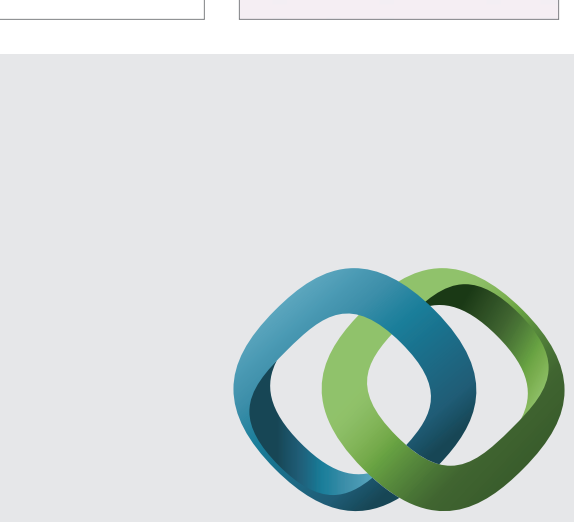

\section{Hindawi}

Submit your manuscripts at

http://www.hindawi.com
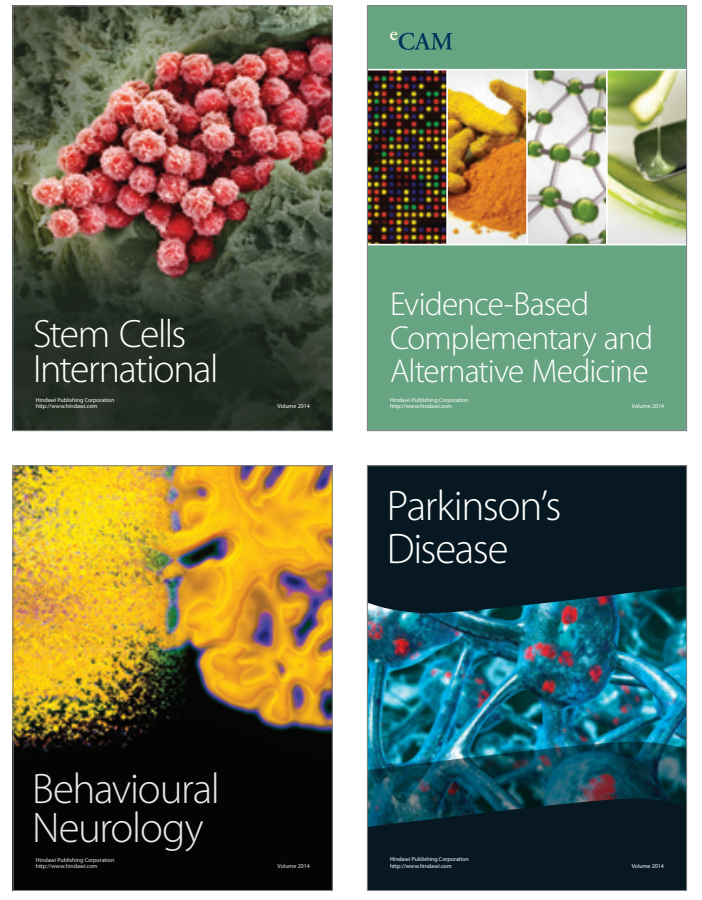
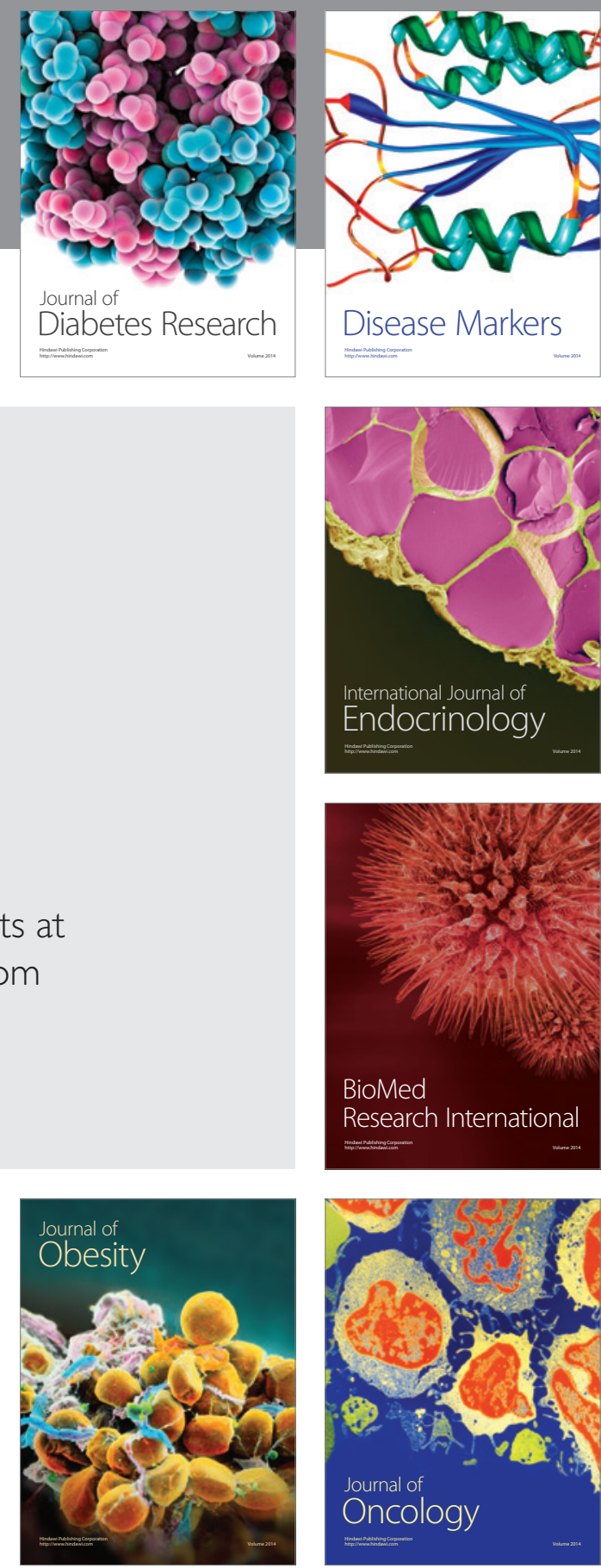

Disease Markers
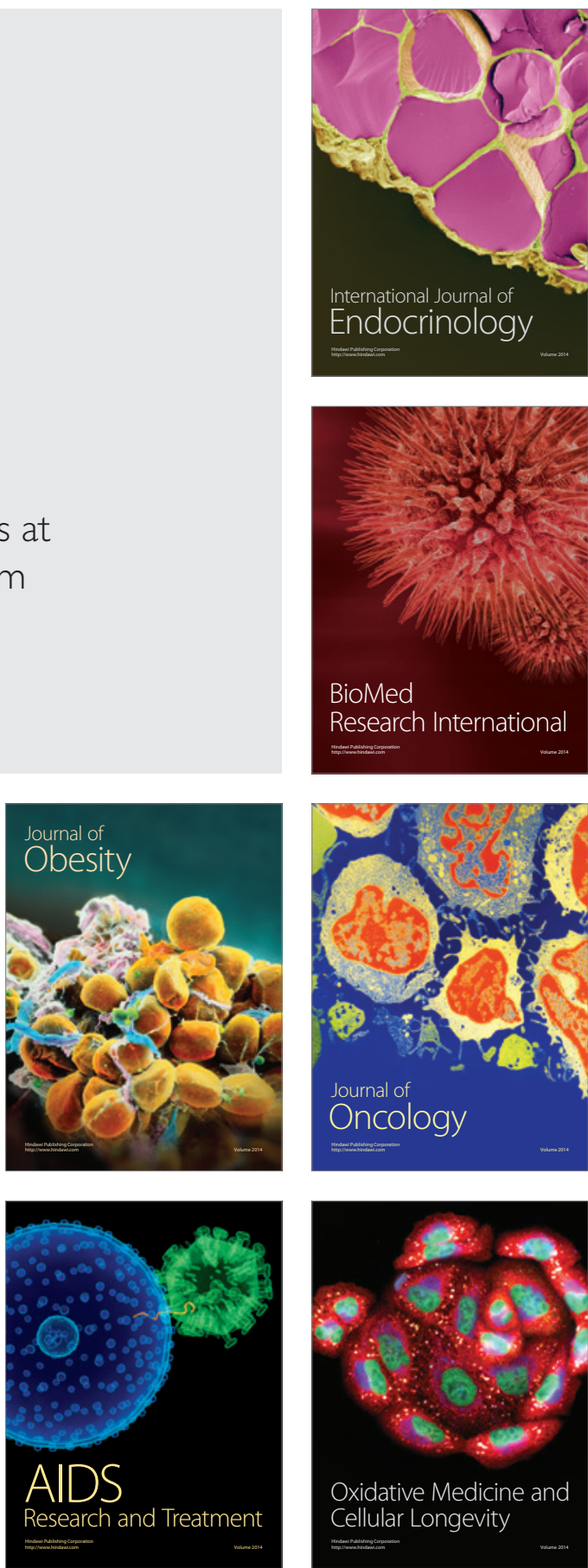